\title{
EL SESGO DE SOBRECONFIANZA EN LAS TAREAS DE IDENTIFICACIÓN Y RECONOCIMIENTO DE ESTIÍMULOS GUSTATIVOS
}

\section{THE OVERCONFIDENCE BIAS IN IDENTIFICATION AND RECOGNITION TASKS OF GUSTATIVE STIMULI}

\author{
Eugenia RaZumiejczyk', Guillermo Macbeth ${ }^{1}$ \\ Consejo Nacional de Investigaciones Cientificas y Técnicas (CONICET), Universidad del Salvador \\ Instituto de InVESTIGACIONES Psicológicas (IIPUS) \\ Buenos Aires, Argentina \\ (RECIBIDO EL 4/02/2009, ACEPTADO EL 24/06/2009)
}

\begin{abstract}
RESUMEN
Se define calibración como la relación entre el éxito objetivo o de rendimiento y el éxito subjetivo o de estimación del éxito alcanzado. En diversos dominios se ha encontrado una tendencia general al sesgo de sobreconfianza, entendido como el predominio del éxito subjetivo por sobre el éxito objetivo. El propósito de este estudio es evaluar la calibración en relación con la modalidad gustativa en las tareas experimentales de identificación y reconocimiento. Los datos mostraron 1) el mismo patrón de sobreconfianza en ambas tareas; 2) que la calibración no se diferenció significativamente de modo que la magnitud del sesgo fue similar. Estos resultados permiten incluir la modalidad gustativa entre los dominios en los que se ha observado el sesgo de sobreconfianza.
\end{abstract}

Palabras clave: Calibración, sesgo de sobreconfianza, gusto, identificación, reconocimiento.

\begin{abstract}
The psychology of thinking has defined calibration as the relation of convergence or divergence between the objective success or actual performance and the subjective success or personal estimation of achievement. The most observed calibration phenomena known as overconfidence bias is defined as the predominance of the subjetive success over the objective success. This calibration bias has been studied in several tasks. The aim of the present study is to extend the state of the art on overconfidence bias in the direction of taste perception. An experiment was conducted to generate this distortion in tasks of identification and recognition of gustative stimuli. Results are consistent with previous studies and generalize the overconfidence bias to the gustative modality.
\end{abstract}

Keywords: Calibration, overconfidence bias, taste, identification, recognition.

1 Investigadores del Consejo Nacional de Investigaciones Científicas y Técnicas (CONICET), Universidad del SalvadorArgentina. E-mail: eugeniaraz@fibertel.com.ar / E-mail: guimacbeth@hotmail.com 


\section{INTRODUCCIÓN}

Uno de los problemas de investigación cognitiva básica que mayor cantidad de aplicaciones ha generado dentro y fuera del laboratorio psicológico es el fenómeno de la calibración C (Kahneman, Slovic \& Tversky, 1982; Kahneman \& Tversky, 2000). Este fenómeno es definido como la relación que existe entre el éxito objetivo $\mathrm{O}$ y el éxito subjetivo $\mathrm{E}$ que presenta una persona en la realización de una colección de n tareas. El éxito objetivo O es entendido como una variable de rendimiento, mientras que el éxito subjetivo E es definido como la creencia personal que el sujeto tiene acerca del éxito objetivo alcanzado. Se admiten tres fenómenos posibles, según se indica en la Ecuación 1 (Gigerenzer, Hoffrage \& Kleinbölting, 1991; Macbeth, Razumiejczyk, Ledesma, Cortada de Kohan \& López Alonso, 2008; Oskamp, 1965).

$$
\begin{array}{ll}
\quad \mathrm{n} & (\mathrm{E}>\mathrm{O})=(\mathrm{C}+)=\text { sesgo de sobreconfianza } \\
\mathrm{C}=\sum_{\mathrm{i}=1} \mathrm{E}_{1}-\mathrm{O}_{1}= & (\mathrm{E}<\mathrm{O})=(\mathrm{C}-)=\text { sesgo de sobreconfianza } \\
& (\mathrm{E}=\mathrm{O})=(\mathrm{C}=\text { cero })=\text { ausencia sesgo de }
\end{array}
$$

Cuando el éxito subjetivo E y el éxito objetivo O coinciden, se considera que la persona se encuentra bien calibrada, es decir, se observa una ausencia de sesgo, por lo cual la calibración C resulta igual a cero en la Ecuación 1. Si, por el contrario, el éxito subjetivo E y el éxito objetivo $\mathrm{O}$ discrepan, se afirma que la calibración $\mathrm{C}$ del sujeto se encuentra sesgada. El sesgo de sobreconfianza se observa cuando el éxito subjetivo E resulta mayor que el éxito objetivo O, por lo cual la calibración $\mathrm{C}$ resulta positiva. En el caso contrario, cuando el éxito subjetivo E resulta menor que el éxito objetivo $O$, se observa el sesgo de subconfianza, es decir, que la calibración $\mathrm{C}$ resulta negativa.

El comportamiento espontáneo de la calibración $\mathrm{C}$ se ha estudiado en diversos dominios de tareas (Kahneman, 2003; Koehler, Brenner \& Griffin, 2002; Lichtenstein, Fischhoff \& Phillips, 1982; Macbeth \& López Alonso, 2008). Se han realizado, por ejemplo, estudios del dominio económico (Angner, 2006; Camerer \& Lovallo, 1999), médico (ChristensenSzalanski \& Bushyhead, 1981), físico (Henrion \& Fischhoff, 1986), psicofísico (Garriga Trillo, Villarino, González Labra \& Arnau, 1994), matemático (Macbeth, Razumiejczyk \& Cortada de Kohan, 2006), verbal (Macbeth \& Cortada de Kohan, 2008; Macbeth \& Razumiejczyk, 2008). Se han llevado a cabo estudios con diversas pruebas, como por ejemplo, definiciones de palabras o la observación de rostros (Johansson \& Allwood, 2007; Kelemen, Frost \& Weaver III, 2000; Klin, Guzmán \& Levine, 1997; Maki, 1999). Sucesivas revisiones del estado de avance de los conocimientos han sostenido que la calibración presenta una propensión general al sesgo de sobreconfianza (Gigerenzer et al., 1991; Koehler et al., 2002; Macbeth \& López Alonso, 2008). En este sentido, Jönsson \& Olsson (2003) y Jönsson, Olsson \& Olsson (2005) hallaron una fuerte sobreconfianza en la habilidad para la identificación de estímulos olfativos.

En tal contexto, el propósito del presente estudio es extender el estado actual de los conocimientos sobre la calibración al dominio gustativo. La hipótesis general de trabajo 
sostiene que las tareas de identificación y reconocimiento de estímulos gustativos generan el sesgo de sobreconfianza, en coherencia con estudios previos aplicados a otras modalidades (Johansson \& Allwood, 2007; Jönsson et al., 2005; Macbeth \& Razumiejczyk, 2008).

Se denomina identificación al proceso cognitivo que se encuentra vinculado al proceso de percepción que organiza los estímulos sensoriales de modo que el participante pueda ser capaz de nombrar el estímulo gustativo que está siendo administrado (Kosslyn \& Rosenberg, 2004). El concepto de reconocimiento consiste, en cambio, en el procesamiento cognitivo que el participante lleva a cabo cuando, luego de la administración de un estímulo gustativo, se le presenta verbalmente el mismo estímulo junto con otros para que reconozca el estímulo gustativo presentado previamente (Baddeley, 1999; Ballesteros \& García Rodríguez, 2000; Düzel, Vargha-Khadem, Heinze \& Mishkin, 2001; Kosslyn \& Rosenberg, 2004).

\section{MÉTODO}

\section{Participantes}

Participaron del estudio 91 estudiantes argentinos reclutados de cursos universitarios de grado. La edad promedio de los participantes resultó de 23,78 años (de $=5,428$ ). La muestra contó con 48 mujeres $(52,7 \%)$ y con 43 varones $(47,3 \%)$. Se establecieron dos grupos aleatorios de participantes, el grupo experimental $1(n=45)$ y el grupo experimental $2(n=46)$. Se mantuvieron proporciones similares de varones y mujeres en ambos grupos. La participación en el experimento fue libre, voluntaria, con conocimiento y consentimiento escrito de cada participante. Los criterios de inclusión en la muestra fueron los siguientes: 1) los participantes debían ser no fumadores, y 2) los participantes no debían haber ingerido ningún alimento ni bebida que no fuera agua durante las tres horas previas al experimento. Estos criterios replican condiciones experimentales empleadas en estudios previos (Razumiejczyk, Macbeth \& Adrover, 2008; López Alonso \& Razumiejczyk, 2008).

\section{Hipótesis}

La hipótesis experimental 1 afirma que el éxito subjetivo $E$ es mayor que el éxito objetivo $O$ en la tarea de identificación $I D E$ de los estímulos gustativos. Formalmente, $H_{1}: \bar{E}_{I D E}>\bar{O}_{I D E}$. Se justifica esta hipótesis por su coherencia con el estado actual de los conocimientos disponibles acerca del sesgo de sobreconfianza observado en diversos dominios (Wilson, Centerbar \& Brekke, 2002). Esta hipótesis se refiere al grupo experimental 1 cuyos participantes deben identificar los ocho estímulos gustativos y luego estimar su número de aciertos.

La hipótesis experimental 2 afirma que el éxito subjetivo $E$ es mayor que el éxito objetivo $O$ en la tarea de reconocimiento $R E C$ de los estímulos gustativos. Formalmente, $H_{2}: \bar{E}_{R E C}>\bar{O}_{R E C}$. Esta hipótesis se refiere al grupo experimental 2 cuyos participantes deben reconocer cada estímulo gustativo administrado sobre la base de una colección de cuatro opciones que se presentan verbalmente, de las cuales una y solo una es la correcta, y al finalizar la prueba, estimar el número de aciertos. Se espera observar el fenómeno 
de sobreconfianza en este grupo experimental por su coherencia con estudios previos (Gigerenzer et al., 1991; Koehler et al., 2002).

Las hipótesis experimentales 1 y 2 se justifican por estudios previos que mostraron la ocurrencia de sobreconfianza en la administración de estímulos olfativos (Jönsson \& Olsson, 2003; Jönsson et al., 2005). Se espera que la calibración en el dominio gustativo se comporte de manera similar a la calibración en el dominio olfativo por tratarse en ambos casos de procesos disparados por estímulos químicos afines (Kosslyn \& Rosenberg, 2004; Razumiejczyk, Macbeth \& López Alonso, 2008c).

La hipótesis experimental 3 afirma que la calibración $C$ no presenta diferencias significativas entre la tarea de identificación $I D E$ del grupo experimental 1 y la tarea de reconocimiento $R E C$ del grupo experimental 2. Formalmente, $H_{3}: \bar{C}_{I D E} \approx \bar{C}_{R E C}$. Se pronostica que el tipo de tarea no influye en el fenómeno de la calibración $C$ de la percepción gustativa.

\section{MÉTODO}

\section{Materiales}

Se emplearon estímulos gustativos naturales y cotidianos en concordancia con su funcionalidad ecológica (Dhami, Hertwig \& Hoffrage, 2004; Gigerenzer et al., 1991; Macbeth \& López Alonso, 2008). Los estímulos utilizados, así como el modo de administración se derivan de estudios previos (Razumiejczyk, Macbeth \& López Alonso, 2008a; Razumiejczyk, Macbeth \& López Alonso, 2008b; Razumiejczyk et al., 2008). Los estímulos administrados fueron brécol, zanahoria, calabacín, calabaza, manzana, durazno, ananá y banana. La etiqueta empleada en este artículo para denominar los estímulos gustativos respeta las normas del Diccionario de la Real Academia Española en su vigésima segunda edición. La presentación de estos estímulos a los participantes se realizó en forma de papilla y a temperatura ambiente. Esta forma de administración intentó prever que no se reconozca el objeto del gusto por su textura y temperatura y, a su vez, que los participantes pudieran efectuar los movimientos naturales de la boca al comer. Asimismo, se empleó un antifaz para evitar que el color de los estímulos influya en su identificación espontánea (Hoegg \& Alba, 2007). Se proporcionó a cada participante una bolsa de polietileno nueva, esterilizada y cerrada, que en su interior contenía un vaso, una servilleta de papel y ocho cucharitas, una para cada estímulo gustativo.

\section{Procedimiento}

Se entrenó a un experimentador voluntario quien no conoció el propósito del estudio hasta finalizada la toma de datos, de modo que funcionó como un experimentador ciego. Sobre un escritorio se ubicaban ocho recipientes tapados, cada uno con un estímulo gustativo. Una vez ingresado el participante al laboratorio, el experimentador constataba que haya cumplido con las condiciones de inclusión, es decir, ser no fumador y no haber ingerido alimentos ni bebidas distintas de agua recientemente. Se le explicaba el procedimiento del experimento enfatizando que la totalidad de los materiales utilizados era desechable. Luego se indicaba al participante que abra la bolsa entregada y retire la servilleta de papel 
y el vaso. El participante debía llenar su vaso con agua que se encontraba disponible sobre el escritorio y se le consignaba que debía beber unos sorbos antes de cada ensayo. Luego se bloqueaba la visión del participante con un antifaz y el experimentador comenzaba la administración sucesiva de los ocho estímulos gustativos. La consigna para los participantes del grupo experimental 1 consistía en identificar el objeto del gusto, es decir, verbalizar el nombre del estímulo gustativo administrado. La consigna para los participantes del grupo experimental 2, en cambio, consistía en reconocer el objeto del gusto a partir de elegir una opción frente a cuatro opciones que se presentaban verbalmente, de las cuales una y solo una era la correcta. Las opciones, así como el orden de las mismas para cada estímulo gustativo fueron aleatorias. Al finalizar la tarea gustativa de identificación o reconocimiento que permitió medir el éxito objetivo $O$, se solicitó a cada participante una estimación subjetiva de éxito para la colección de estímulos que permitió medir la variable de éxito subjetivo $E$. Cada sujeto debió estimar cuántos de los ocho estímulos administrados creyó haber acertado. Finalmente, el experimentador agradecía al participante la colaboración y lo acompañaba a la puerta de salida. Se arrojaban al cesto de residuos el vaso descartable y la servilleta de papel utilizados. El orden de la administración de los estímulos para cada participante fue aleatorio.

\section{RESULTADOS}

La hipótesis $H_{1}$ resultó coherente con la evidencia experimental. La media de éxito subjetivo para el grupo experimental $\underline{1}$ resultó de $\bar{E}_{I D E}=4,91(d e=1,649)$, mientras que la media de éxito objetivo fue de $\bar{O}_{I D E}=3,96(d e=1,429)$. Con el fin de comparar ambas variables, se realizó una prueba $t$ para muestras apareadas que mostró una diferencia estadísticamente significativa $(t=6,142 ; p<0,01 ; d$ de Cohen $=0,62)$. Este resultado muestra la ocurrencia del sesgo de sobreconfianza en el grupo experimental 1.

La hipótesis $\mathrm{H}_{2}$ obtuvo evidencia favorable. La media de éxito subjetivo para el grupo experimental 2 resultó de $\bar{E}_{R E C}=4,91(d e=1,226)$, mientras que la media de éxito objetivo fue de $\bar{O}_{R E C}=4,15(d e=1,49)$. La comparación de estas variables mediante la prueba $t$ para muestras apareadas mostró una diferencia significativa $(t=5,318 ; p<0,01$; $d$ de Cohen =0,56). Este resultado permite afirmar que el grupo experimental 2 incurrió en el sesgo de sobreconfianza.

La hipótesis $H_{3}$ resultó asimismo coherente con la evidencia experimental. La media de la calibración para el grupo experimental 1 resultó de $\bar{C}_{I D E}=0,96(d e=1,043)$, mientras que la media de la calibración para el grupo experimental 2 fue de $\bar{C}_{R E C}=0,76(d e=0,97)$. Con el fin de comparar estas variables, se realizó una prueba $t$ para muestras independientes que mostró una diferencia no significativa $(t=0,922 ; p=0,359 ; d$ de Cohen $=0,19)$. Este resultado indica que el sesgo de sobreconfianza presentó una magnitud similar en ambos grupos experimentales.

Si bien se esperaba que las pistas facilitadas al grupo experimental 2 en la tarea de reconocimiento generen ciertas ventajas sobre el grupo experimental 1, no se observó una diferencia significativa al comparar el éxito objetivo $O$ en ambos grupos $(t=-0,642$; $p=0,522 ; d$ de Cohen $=0,13$ ). Las pistas no facilitaron la tarea de reconocimiento en comparación con las tareas de identificación. 
La Tabla N. ${ }^{\circ} 1$ resume los puntajes de calibración obtenidos y la Figura N. ${ }^{\circ} 1$ ofrece una representación gráfica de la relación entre las variables $E, O$ y $C$ ante tareas de identificación y reconocimiento de los estímulos gustativos.

Todas las variables incluidas en las pruebas de hipótesis resultaron normales y homocedásticas por las pruebas de Kolmogorov-Smirnov y Levene, respectivamente.

Tabla N. ${ }^{0}$ 1. Calibración en identificación y reconocimiento de estímulos gustativos.

\begin{tabular}{lcc}
\hline & \multicolumn{2}{c}{ Grupo experimental } \\
\cline { 2 - 3 } Tarea gustativa & Identificación & \multicolumn{1}{c}{2} \\
\cline { 2 - 3 } Éxito subjetivo $\bar{E}(\boldsymbol{d})$ & $5,67(1,384)$ & $6,04(0,934)$ \\
Éxito objetivo $\bar{O}(\boldsymbol{d})$ & $4,23(1,25)$ & $3,85(1,713)$ \\
Calibración $\bar{C}(\boldsymbol{d})$ & $0,96(1,043)$ & $0,76(0,97)$ \\
Calibración observada & Sesgo de sobreconfianza & Sesgo de sobreconfianza \\
\hline
\end{tabular}

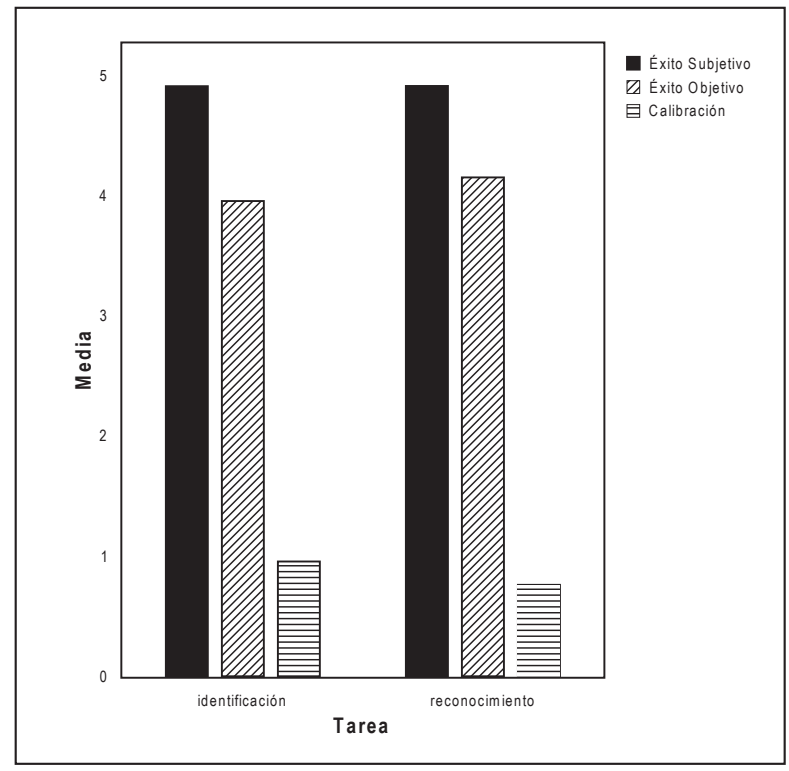

Figura N. ${ }^{0}$ 1. Comparación entre el éxito subjetivo, el éxito objetivo y la calibración en dos tareas gustativas. 


\section{DISCUSIÓN}

Los resultados mostraron la ocurrencia del sesgo de sobreconfianza tanto en las tareas de identificación consignadas al grupo experimental 1, como en las tareas de reconocimiento solicitadas al grupo experimental 2. Así, en ambas tareas gustativas se observó que el éxito subjetivo $E$ resultó significativamente mayor que el éxito objetivo $O$. Estos datos se encuentran en concordancia con estudios previos (Jönsson \& Olsson, 2003; Jönsson et $a l .$, 2005) que mostraron la ocurrencia del sesgo de sobreconfianza en la identificación de estímulos olfativos. No obstante, este estudio mostró que el fenómeno de sobreconfianza se produjo en dos pruebas diferentes aunque relativas a la modalidad gustativa, esto es, la identificación y el reconocimiento. Asimismo, los datos mostraron que la calibración no se diferenció significativamente entre ambas tareas de modo que, si bien ambos grupos experimentales sobreconfiaron sus resultados, la magnitud del sesgo fue similar.

Los estímulos químicos olfativos y gustativos son considerados afines (Razumiejczyk et al., 2008c), por lo cual resulta razonable que ambos presenten un mismo patrón de calibración. En este sentido, resultaría esperable la observación del sesgo de sobreconfianza dado que es coherente con la seguridad en el acierto subjetivo por la fortaleza del fenómeno perceptivo. Este resultado, sin embargo, pudo ser diferente entre ambas modalidades debido a que los fenómenos de la calibración son de dominio específico (Macbeth \& Morán, en prensa). Se espera que tareas afines generen patrones de calibración también afines, pero no de manera necesaria o sistemática porque cada dominio posee sus propias tendencias de calibración (Kelemen et al., 2000; Macbeth, Cortada de Kohan, Razumiejczyk \& López Alonso, 2006; Macbeth \& Fernández, en prensa).

Una limitación del presente estudio consiste en que se propuso sólo describir el sesgo de sobreconfianza, pero no reducirlo como lo sugieren diversas líneas actuales de investigación sobre estos fenómenos (Macbeth \& Razumiejczyk, 2008). Se recomienda emplear en futuras investigaciones entrenamientos metacognitivos en calibración para intentar reducir el sesgo de sobreconfianza en la identificación y el reconocimiento de estímulos gustativos. Esta estrategia ha resultado exitosa en estudios previos aplicados a otras modalidades.

\section{REFERENCIAS BIBLIOGRÁFICAS}

1. Angner, E. (2006). Economists as experts: Overconfidence in theory and practice. Journal of Economic Methodology, 13(1), 1-24.

2. Baddeley, A. (1999). Memoria humana. Teoría y práctica. Barcelona: McGrawHill.

3. Ballesteros, S. \& García Rodríguez, B. (2000). Procesos psicológicos básicos. Editorial Universitas: España.

4. Camerer, C.F. \& Lovallo, D. (1999). Overconfidence and excess entry: An experimental approach. American Economic Review, 89(1), 306-318.

5. Christensen-Szalanski, J.J. \& Bushyhead, J.B. (1981). Physicians' use of probabilistic information in a real clinical setting. Journal of Experimental Psychology: Human perception and performance, 7, 928-935. 
6. Dhami, M.K., Hertwig, R. \& Hoffrage, U. (2004). The role of representative design in an ecological approach to cognition. Psychological Bulletin, 6, 959-988.

7. Düzel, E., Vargha-Khadem, F., Heinze, H.J. \& Mishkin, F. (2001). Brain activity evidence for recognition without recollection after early hippocampal damage. Proceedings of the National Academy of Sciences, 98(14), 8101-8106.

8. Garriga Trillo, A.J., Villarino, A., González Labra, M.J. \& Arnau, M.A. (1994). La calibración de juicios psicofísicos: Estimación de magnitudes. Psicothema, 6(3), 525-532.

9. Gigerenzer, G., Hoffrage, U. \& Kleinbölting, H. (1991). Probabilistic mental models: A brunswikian theory of confidence. Psychological Review, 98(4), 506-528.

10. Henrion, M. \& Fischhoff, B. (1986). Assessing uncertainty in physical constants. American Journal of Physics, 54, 791-797.

11. Hoegg, J. \& Alba, J.W. (2007). Taste perception: more than meets the tongue. Journal of Consumer Research, 33(4), 490-498.

12. Johansson, M. \& Allwood, C.M. (2007). Cognition and neurosciences. Scandinavian Journal of Psychology, 48, 13-21.

13. Jönsson, F.U. \& Olsson, M.J. (2003). Olfactory metacognition. Chemical Senses, $28,651-658$.

14. Jönsson, F.U., Olsson, H. \& Olsson, M.J. (2005). Odor emotionality affects the confidence in odor naming. Chemical Senses, 30, 29-35.

15. Kahneman, D. (2003). Perspective on judgment and choice. Mapping bounded rationality. American Psychologist, 58 (9), 697-720.

16. Kahneman, D. \& Tversky, A. (Eds.). (2000). Choices, Values, and Frames. New York: Cambridge University Press.

17. Kahneman, D., Slovic, P. \& Tversky, A. (Eds.). (1982). Judgment under uncertainty: Heuristics and biases. Cambridge: Cambridge University Press.

18. Kelemen, W.L., Frost, P.J. \& Weaver III, C.A. (2000). Individual differences in metacognition: evidence against a general metacognitive ability. Memory \& Cognition, 28, 92-107.

19. Klin, C.M., Guzmán, A.E. \& Levine W.H. (1997). Knowing that you don't know: metamemory and discourse processing. Journal of Experimental Psychology, 23, 1378-1393.

20. Koehler, D.J., Brenner, L. \& Griffin, D. (2002). The Calibration of expert judgment: Heuristics and biases beyond the laboratory. En T. Gilovich, D. Griffin \& D. Kahneman (Eds.). Heuristics and Biases. The psychology of intuitive judgment (pp. 686-715). Cambridge: Cambridge University Press.

21. Kosslyn, S. \& Rosenberg, R. (2004). Psychology. The brain, the person, the world. Estados Unidos: Pearson. 
22. Lichtenstein, S., Fischhoff, B. \& Phillips, L.D. (1982). Calibration of probabilities: The state of the art to 1980. En D. Kahneman, P. Slovic \& A. Tversky (Eds.). Judgment under uncertainty: Heuristics and biases (pp. 306-334). Cambridge: Cambridge University Press.

23. López Alonso, A.O. \& Razumiejczyk, E. (2008). Evidencia de priming gustativo en condición de codificación superficial y profunda y su disociación con la memoria explícita. Perspectivas en Psicología, 5(1), 50-57

24. Macbeth, G. \& Fernández, H. (en prensa). Moderadores metacognitivos del sesgo de subconfianza. Informes Psicológicos (Medellín).

25. Macbeth, G. \& Morán, V. (en prensa). El sesgo de subconfianza como fenómeno de dominio específico. Revista Latinoamericana de Psicología.

26. Macbeth, G., Cortada de Kohan, N., Razumiejczyk, E. \& López Alonso, A. O. (2006). Los sesgos de sobreconfianza y subconfianza en tareas de conocimientos generales. Acta Psiquiátrica y Psicológica de América Latina, 52, 221-226.

27. Macbeth, G., Razumiejczyk, E. \& Cortada de Kohan, N. (2006). El sesgo de sobreconfianza en tareas verbales y matemáticas. Investigaciones en Psicología UBA, 11(3), 47-58.

28. Macbeth, G. \& Cortada de Kohan, N. (2008). Efecto del entrenamiento sobre la calibración subjetiva de éxito en tareas verbales. Revista Latinoamericana de Psicología, 40(1), 9-20.

29. Macbeth, G. \& López Alonso, A.O. (2008). Aportes de enfoque ecológico a los estudios sobre calibración. Acta Psiquiátrica y Psicológica de América Latina, 54(1), 55-61.

30. Macbeth, G. \& Razumiejczyk, E. (2008). Disolución del sesgo de subconfianza en tareas verbales. Anales de Psicología, 24(1), 143-149.

31. Macbeth, G., Razumiejczyk, E., Ledesma, R., Cortada de Kohan, N. \& López Alonso, A.O (2008). La medición de los sesgos de la confianza mediante modelos discretos y continuos. Investigaciones en Psicología, 13, 117-134.

32. Maki, R.H. (1999). The roles of competition, target accessibility, and cue familiarity in metamemory for word pairs. Journal of Experimental Psychology, 25, 1011-1023.

33. Oskamp, S. (1965). Overconfidence in case-study judgments. The Journal of Consulting Psychology, 29, 261-265.

34. Razumiejczyk, E., Macbeth, G. \& Adrover, J.F. (2008). Priming intramodal e intermodal: un estudio gustativo-visual. Revista de Investigación en Psicología, 11(1), 69-79.

35. Razumiejczyk, E., Macbeth, G. \& López Alonso, A.O. (2008a). Evidencia de priming gustativo y su disociación con la memoria explícita. Revista Universitas Psychologica, $7(2), 549-556$. 
36. Razumiejczyk, E., Macbeth, G. \& López Alonso, A.O. (2008b). Efecto de priming gustativo en condición de codificación profunda y su disociación con la memoria explícita. Investigaciones en Psicología, 13(3), 45-56.

37. Razumiejczyk, E. Macbeth, G. \& López Alonso (2008c). La vinculación entre las modalidades gustativa y olfativa en el reconocimiento del sabor. Psico Logos, 17, 5-12.

38. Wilson, T.D., Centerbar, D.B. \& Brekke, N. (2002). Mental contamination and the debiasing problem. En T. Gilovich, D. Griffin \& D. Kahneman (Eds.), Heuristics and Biases. The psychology of intuitive judgment (pp. 185-200). Cambridge: Cambridge University Press. 